\title{
SUBJECTIVE EXPECTED UTILITY WITH NONINCREASING RISK AVERSION
}

\author{
Peter P. WAKKER * \\ Department of Mathematical Psychology, University of Nijmegen (NICI), \\ P.O. Box 9104, NL-6500 HE Nijmegen, The Netherlands
}

\begin{abstract}
It is shown that assumptions about risk aversion, usually studied under the presupposition of expected utility maximization, have a surprising extra merit at an carlier stage of the measurement work: together with the sure-thing principle, these assumptions imply subjective expected utility maximization for monotonic continuous weak orders.
\end{abstract}

\section{Introduction}

There has not yet been obtained a characterization of subjective expected utility maximization in the literature that is fully satisfactory in the sense of using necessary and sufficient conditions that are all empirically meaningful and have a clear intuitive meaning. The main nonnecessary condition used by Savage [7] is his P6, a kind of continuity condition, requiring some structure on the state space, which must be "fine". The main nonnecessary condition used in Wakker [9-11] is a continuity condition, requiring structure on the consequence space, which also must be "fine" in some sense.

Because of the frequent use of subjective expected utility models and all kinds of special forms of these, further "derivations" seem desirable. A derivation of a (specified form of a) subjective expected utility model gives a list of conditions sufficient for the applicability of the model. It is desirable that the list be as short as possible, with as many as possible behaviourally meaningful conditions (such as Savage's $\mathrm{P} 3$, the "sure-thing principle", and his $\mathrm{P} 4$, ensuring the existence of a "more probable than" relation; or Wakker's "cardinal coordinate independence", a strengthening of the sure-thing principle), with few technical conditions (such as the "Archimedean

\footnotetext{
"This study was carried out while the author was at the Netherlands Central Bureau of Statistics, Department of Statistical Methods, Voorburg, The Netherlands.
}

c) J.C. Baltzer AG, Scientific Publishing Company 
axioms" in Krantz et al. [5], see Adams et al. [1]), and with as few conditions as possible that have an unclear empirical status (such as P6 in Savage [7], and continuity in Wakker $[9-11])$.

In this paper we shall restrict attention to preference relations of decision makers who face uncertainty about the monetary consequences of possible actions. We shall derive, for finite state spaces, the subjective expected utility model with risk aversion and nonincreasing (or nondecreasing; or constant; or constant relative) risk aversion. It will be shown that these assumptions on risk aversion simplify the derivation of subjective expected utility maximization by making superfluous Savage's P4 or Wakker's cardinal coordinate independence strengthening of the sure-thing principle. The only nonbehavioural and nonnecessary condition that we shall use is continuity of the preference relation, amounting to continuity of the utility function.

The proof of our main result, theorem 3.3 , is based on the adaption of theorems of Pratt [6] to the case of continuous (not necessarily differentiable) utility functions, and to the context of decision making under uncertainty (without all probability distributions available), as it has been given in Wakker et al. [12]; on a theorem of Debreu [2] for additively decomposable representing functions; and on a theorem of Debreu and Koopmans [3] on differentiability properties of additively decomposable quasiconvex functions.

Subsequent theorems use a result of Stehling [8] on additively decomposable homothetic functions.

\section{Decision making under uncertainty}

Let $S=\left\{s_{1}, \ldots, s_{n}\right\}$ denote a finite state space, elements of which are (possible) states (of nature). Exactly one is the true state, the others are untrue. By $G$ we denote a nondegenerate interval, i.e. a convex subset of $\mathbb{R}$ with more than one, so uncountably many, elements. Elements of ' $\mathrm{C}$ are consequences, and are interpreted as amounts of money. Elements of $\iota_{C}^{n}$ are called acts. An act $x=\left(x_{1}, \ldots, x_{n}\right)$ yields consequence $x_{j}$ if state $j$ is the true state.

Let $\xi$ be a binary relation on $\epsilon^{n}$ that denotes the preference relation of a decision maker who is uncertain about which state of nature is the true one. We write $x \leq y$ for $y \leqq x, x \approx y$ for $[x \xi y$ and $y \xi x], x \varepsilon y$ for $[x \xi y$ and not $y \xi x], x-y$ for $y \& x$. We call $\leqq$ a weak order if it is complete ( $x \underline{\xi} y$ or $y \xi x$ for all $x, y$ ) and transitive. Further, $\subseteq$ is continuous if $\{x: x \underline{\varepsilon} y\}$ and $\{x: x \leqq y\}$ are closed for all $y$, and $\_$is convex if $\{x: x \leqq y\}$ is convex for every $y$. In this paper, $\xi$ will always be strongly monotonic, i.e. $x \& y$ whenever $x_{j} \geqslant y_{j}$ for all $j$, and $x \neq y$. This implies that no state of nature is considered impossible, and that all coordinates are "essential" (see definition 2.8), i.e. have influence on the preference relation.

A function $V: \mathscr{C}^{n} \rightarrow \mathbb{R}$ represents $\underline{\varepsilon}$ if $[x \underline{\varepsilon} \Leftrightarrow V(x) \geqslant V(y)]$ for all $x, y \in \mathcal{C}^{n} . V$ is additively decomposable if there exist $V_{j}: G \rightarrow \mathbb{R}, j=1, \ldots, n$, 
such that $V(x)=\Sigma V_{j}\left(x_{j}\right)$ for all $x$. Such $V_{j}$ 's are called additive value functions

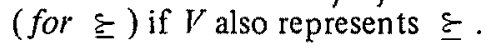

NOTATION 2.1

For $1 \leqslant i \leqslant n, x \in \ell^{n}, \alpha \in \mathcal{G}, x_{-i} \alpha$ is $\left(x\right.$ with $x_{i}$ replaced by $\alpha$ ). If further $1 \leqslant j \leqslant n, j \neq i, \beta \in \ell^{\prime}$, then $\left(x_{-i, j} \alpha, \beta\right)$ is $\left(x\right.$ with $x_{i}$ replaced by $\alpha, x_{j}$ by $\left.\beta\right)$.

NOTATION 2.2

For $\alpha \in \in^{\prime} \in, \bar{\alpha}$ is $(\alpha, \alpha, \ldots, \alpha)$.

\section{DEFINITION 2.3} $\alpha, \beta \in \mathcal{C}^{\prime}:$

We say $\leqq$ satisfies the sure-thing principle if for all $x, y \in \ell^{n}, 1 \leqslant i \leqslant n$,

$$
x_{-i} \alpha \underline{\xi} y_{-i} \alpha \Leftrightarrow x_{-i} \beta \underline{\varepsilon} y_{-i} \beta .
$$

One can see that $£$ satisfies the sure-thing principle if and only if: $[x £ y \Leftrightarrow v £ w]$ whenever for each $i$ either $\left[x_{i}=v_{i}\right.$ and $\left.y_{i}=w_{i}\right]$ or $\left[x_{i}=y_{i}\right.$ and $\left.v_{i}=w_{i}\right]$. This latter condition is derived from the sure-thing principle by (finiteness of $S$ and) substitution, one by one, of $v_{i}$ and $w_{i}$ for $x_{i}$ and $y_{i}$, for all those $i$ for which $x_{i}=y_{i}, v_{i}=w_{i}$, and by application of the sure-thing principle after every substitution. The second formulation of the sure-thing principle is the one used in Savage [7].

\section{DEFINITION 2.4}

We call $\left[G^{n}, \subseteq,\left(p_{j}\right)_{j=1}^{n}, U\right]$ a subjective expected utility (SEU) model (for $\underline{\xi}$ ) if the $p_{j}$ 's are nonnegative real numbers, summing to 1 , and $U$ is a function from $\ell_{i}$ to $\mathbb{R}$, such that $\varepsilon$ is represented by the function $V:{ }^{\prime} G^{n} \rightarrow \mathbb{R}$, defined by $V(x):=\sum_{j=1}^{n} p_{j} U\left(x_{j}\right)$ for all $x$.

We then call $p_{j}$ the (subjective) probability for state $s_{j}, U$ the (subjective) utility function, and $V(x)$ the (subjective) expected utility of $x$. $x \in \ell^{n}$ :

For a given SEU model $\left[\complement^{n}, \underline{\varepsilon},\left(p_{j}\right)_{j=1}^{n}, U\right], \underline{\xi}$ is risk averse if, for all

$$
x \preceq\left(\sum_{j=1}^{n} p_{j} x_{j}, \ldots, \sum_{j=1}^{n} p_{j} x_{j}\right) .
$$

It should be noted that this property requires the probabilities to be known. We have the following well-known characterization: 


\section{THEOREM 2.5}

Let $\left[\mathscr{C}^{n}, \underline{\varepsilon},\left(p_{j}\right)_{j=1}^{n}, U\right]$ be an SEU model for $\xi$, with $n \geqslant 2, U$ continuous, and $\underline{\xi}$ strictly monotonic. Then the following three statements are equivalent:

(i) " $U$ is concave".

(ii) " $\underline{\varepsilon}$ is risk averse".

(iii) "s is convex".

The properties introduced below usually are considered only in the presence of risk aversion. We prefer to introduce them in general.

\section{DEFINITION 2.6}

The preference relation $\underline{E}$ has nonincreasing [respectively, nondecreasing; or constant] (absolute) risk aversion if for all $\epsilon \geqslant 0$ [respectively, $\epsilon \leqslant 0$; or $\epsilon \in \mathbb{R}$ ], and for all $x, x+\bar{\epsilon}$ in $\mathscr{C}^{n}, \alpha, \alpha+\epsilon \in \mathscr{G}$, we have:

$$
x \underline{\alpha} \Rightarrow x+\bar{\epsilon} \underline{\varepsilon} \bar{\alpha}+\bar{\epsilon} .
$$

\section{DEFINITION 2.7}

The preference relation $\subseteq$ has constant relative risk aversion if for all $\lambda \in \mathbb{R}_{++}$, $x, \lambda x \in \mathscr{G}^{n}, \alpha, \lambda \alpha \in \mathscr{G}$, we have:

$$
x \leqq \bar{\alpha} \Rightarrow \lambda x \leqq \lambda \bar{\alpha} .
$$

The latter property, while conceptually different, can formally be identified with the condition of "homotheticity" from consumer demand theory. The following property will be used only in reference to other papers:

\section{DEFINITION 2.8}

Coordinate (or index) $i$ is essential if there exist $x, \alpha$ such that $x_{-i} \alpha \neq x$.

\section{Subjective expected utility for nonincreasing or nondecreasing risk aversion}

In the lemma below, $V^{\mathrm{r}}(\alpha)$ [respectively, $V^{\ell}(\alpha)$; or $V^{\prime}(\alpha)$ ] denotes the rightderivative [respectively, the left-derivative; or the derivative] in $\alpha$ of a function $V$. 


\section{LEMMA 3.1}

Let $n \geqslant 2$. Let $V_{1}, \ldots, V_{n}$ be continuous strictly increasing additive value functions for $\underline{\varepsilon}$. Let $\underline{\varepsilon}$ be convex, and have nonincreasing risk aversion. Let $\alpha \in$ int ( $(b), \beta \in$ int $(\mathfrak{b}), \alpha>\beta$. Let $i \neq j$. Then:

$$
V_{i}^{\mathrm{r}}(\alpha) V_{j}^{\ell}(\beta) \geqslant V_{j}^{\ell}(\alpha) V_{i}^{\mathrm{r}}(\beta)
$$

whenever the derivatives are defined.

Proof

We take $\left(\sigma^{k}\right)_{k=1}^{\infty},\left(\tau^{k}\right)_{k=1}^{\infty} \in \mathbb{R}_{++}$such that: $\sigma^{k} \downarrow 0, \tau^{k} \downarrow 0, \alpha+\tau_{k}$ and $\beta-\sigma_{k}$ are in $\mathscr{C}$ for all $k, V_{i}\left(\beta+\tau^{k}\right)-V_{i}(\beta)=V_{j}(\beta)-V_{j}\left(\beta-\sigma_{k}\right)$ for all $k$. Such $\sigma^{k}$ and $\tau^{k}$ exist by continuity and strict increasingness of $V_{i}$ and $V_{j}$. For all $k$, it follows that

$$
\left(\bar{\beta}_{-i, j}\left(\beta+\tau^{k}\right),\left(\beta-\sigma^{k}\right)\right) \approx \bar{\beta} .
$$

By nonincreasing risk aversion, it follows that:

$$
\left(\bar{\alpha}_{-i, j}\left(\alpha+\tau^{k}\right),\left(\alpha-\sigma^{k}\right)\right) \underline{\alpha}
$$

i.e.,

$$
V_{i}\left(\alpha+\tau^{k}\right)-V_{i}(\alpha) \geqslant V_{j}(\alpha)-V_{j}\left(\alpha-\sigma^{k}\right)
$$

for all $k$. We obtain:

$$
\begin{aligned}
V_{i}^{\mathrm{r}}(\alpha) V_{j}^{\ell}(\beta) & =\lim _{k \rightarrow \infty}\left[V_{i}\left(\alpha+\tau^{k}\right)-V_{i}(\alpha)\right]\left[V_{j}(\beta)-V_{j}\left(\beta-\sigma^{k}\right)\right] / \tau^{k} \sigma^{k} \\
& \geqslant \lim _{k \rightarrow \infty}\left[V_{j}(\alpha)-V_{j}\left(\alpha-\sigma^{k}\right)\right]\left[V_{i}\left(\beta+\tau^{k}\right)-V_{i}(\beta)\right] / \sigma^{k} \tau^{k}=V_{j}^{\ell}(\alpha) V_{i}^{\mathrm{r}}(\beta) .
\end{aligned}
$$

\section{REMARK 3.2}

If one replaces nonincreasing risk aversion in the above lemma by nondecreasing risk aversion, then

$$
V_{i}^{\mathrm{r}}(\alpha) V_{j}^{\ell}(\beta) \leqslant V_{j}^{\ell}(\alpha) V_{i}^{\mathrm{r}}(\beta)
$$

The proof is analogous to the one above.

We are now ready for our main theorem. 


\section{THEOREM 3.3}

Let $n \geqslant 3$. The following three statements are equivalent for the nondegenerate interval $G$ and the preference relation $\varepsilon$ on $\mathscr{G}^{n}$ :

(i) "There exists an SEU model $\left[G^{n}, \varepsilon,\left(p_{j}\right)_{j=1}^{n}, U\right]$ for $\xi$, with all $p_{j}>0$, and with $U$ continuous, strictly increasing, concave; furthermore, for all $\alpha \geqslant \beta \geqslant \gamma>\delta$ in 6 , the function

$\epsilon \rightarrow[U(\alpha+\epsilon)-U(\beta+\epsilon)] /[U(\gamma+\epsilon)-U(\delta+\epsilon)]$

is nondecreasing on its domain".

(ii) "There exists an SEU model $\left[f^{n}, \xi,\left(p_{j}\right)_{j=1}^{n}, U\right]$ for $\xi$, with all $p_{j}>0, U$ continuous, strictly increasing. Further, $\underline{\varepsilon}$ is risk averse, and has nonincreasing risk aversion".

(iii) "The preference relation $\underline{\xi}$ is a continuous strongly monotonic weak order, it satisfies the sure-thing principle, is convex, and has nonincreasing risk aversion".

\section{Proof}

Part of the equivalence of (i) and (ii) follows from theorem 2.5. We concentrate on the remainder of the equivalence of (i) and (ii). This does not follow from Pratt ([6], mainly (e) in theorem 1), firstly because his $U$ is assumed twice continuously differentiable, and secondly because his context is decision making under risk, with all probability distributions available, whereas we have only a fixed and finite number of probabilities $p_{1}, \ldots, p_{n}$ available. The only result in the literature known to us from which (i) $\Leftrightarrow$ (ii) can be derived is Wakker et al. ([12], theorem 4.1 and lemma A.7.4), mainly by comparing $\leqq$ with $\xi^{\prime}$, defined by $x \xi^{\prime} y$ if $x-\bar{\epsilon} \xi y-\bar{\epsilon}$, on an appropriate domain. For brevity, we omit elaboration. derive (ii).

The implication (ii) $\Rightarrow$ (iii) is straightforward, so we finally assume (iii) and

By strong monotonicity, all (so at least three) coordinates are essential. Since $\xi$ is a continuous weak order, satisfying the sure-thing principle, theorem 3 of Debreu [2] implies that there exist continuous additive value functions $\left(V_{j}\right)_{j=1}^{n}$ for $\varepsilon$. Further, we may assume that for some arbitrary fixed $\mu^{0} \in \mathscr{G}, V_{i}\left(\mu^{0}\right)=0$ for all $j$. By strong monotonicity, the $V_{j}$ 's are strictly increasing. The main problem will now be to show:

The $V_{i}$ s are proportional.

To see this, first we note that the function $V$, assigning $\sum_{j=1}^{n} V_{j}\left(x_{j}\right)$ to every $x$, represents a convex $\underline{\xi}$, and hence is quasiconcave. It now follows easily from the $n$-dimen- 
sional analogue of theorem 3 in Debreu and Koopmans [3] that, for all $i$, on int $\left(\varphi_{6}\right)$ : $V_{i}^{\mathrm{r}}$ and $V_{i}^{\ell}$ are defined and finite, $V_{i}^{\mathrm{T}}$ is continuous on the right, $V_{i}^{l}$ is continuous on the left, and at every point outside a countable set $E_{i}, V_{i}$ is differentiable.

Let $E=U E_{i}$, so $E$ is countable. On [int $\left.(\mathscr{\ell})\right] \backslash E$, all $V_{i}$ are differentiable. Hence, two applications of lemma 3.1 give, for all $i, j$ :

$$
V_{i}^{\prime}(\alpha) V_{j}^{\prime}(\beta)=V_{j}^{\prime}(\alpha) V_{i}^{\prime}(\beta) \text { for all } \alpha, \beta \in[\operatorname{int}(\mathscr{G})] \backslash E \text {. }
$$

It follows from this that there exist $j$ and real numbers $\left(\sigma_{i}\right)_{i=1}^{n}$ such that $V_{i}^{\prime}=\sigma_{i} V_{i}^{\prime}$ on [int ( $\left.\left.{ }^{(} E\right)\right] \backslash E$ for all $i$. Since $E$ is countable and $V_{i}{ }^{\mathrm{r}}, V_{i}^{\mathrm{r}}$ are continuous on the right

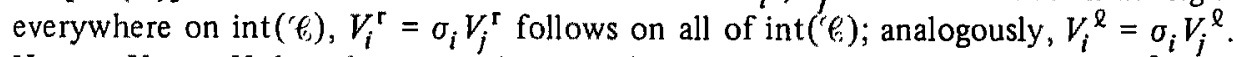
Hence, $V_{i}-\sigma_{i} V_{j}$ has derivative 0 on int( $\left.{ }^{\prime} G\right)$, and so is constant. Since $V_{i}\left(\mu^{0}\right)=0$ $=V_{j}\left(\mu^{0}\right)$, the constant is zero, so continuity yields $V_{i}=\sigma_{i} V_{j}$ on all of ' $\ell$, for all $i$ :

The $V_{i}$ 's indeed are proportional.

By strict increasingness of the $V_{i}$ 's, all $\sigma_{i}$ 's are positive. We define $U:=\left(\sum_{k=1}^{n} \sigma_{k}\right) V_{j}$ and $p_{i}:=\sigma_{i} / \sum_{k=1}^{n} \sigma_{k}$. This gives an SEU model for $\underline{\xi}$. Since, by theorem 2.5 , convexity of $\xi$ implies risk aversion, everything of (ii) follows.

In (iii) above, we have given a characterization of the quantitative model in (i), completely in terms of properties directly in terms of the preference relation $\underline{\xi}$. Hence, we did not use the property of risk aversion in (iii), as this needs the probabilities in its definition. In the context of decision making under uncertainty (unlike the context of decision making under risk), probabilities are not primitives.

One can replace nonincreasing risk aversion by nondecreasing risk aversion in (ii) and (iii) above if one replaces nondecreasingness of the function defined in (3.3) by nonincreasingness. Analogously, one can of course substitute "constant risk aversion" in (ii) and (iii), and constantness of the function defined in (3.3). In the latter case, either $U$ is affine or exponential, as can be derived from theorem 3.5 in

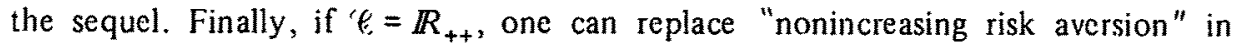
(ii) and (iii) above by "constant relative risk aversion" if one replaces the statement on the function defined in (3.3) by the statement that $U: \alpha \rightarrow \log \alpha$, or $U: \alpha \rightarrow \lambda \alpha^{\rho}$, as can be derived from theorem 3.4 in the sequel.

For theorem 3.3, we do not need the assumption of concavity in (i), of risk aversion in (ii), and of convexity in (iii) if ' $\ell_{2}=\mathbb{R}$ and we havc constant absolute risk aversion, or if $\%=\mathbb{R} R_{++}$and we have constant relative risk aversion. First, we give the latter result, this being directly derivable from Stchling [8] . 


\section{THEOREM 3.4}

Let $n \geqslant 3$. Let $6=\mathbb{Z} R_{++}$. The following two statements are equivalent for the preference relation $\underline{\xi}$ on $\mathscr{C}^{n}$ :

(i) "There exists an SEU model $\left[{ }^{\prime} \ell^{n}, \underline{\xi},\left(p_{j}\right)_{j=1}^{n}, U\right]$ for $\underline{\xi}$, with all $p_{j}>0$, and either $U: \alpha \rightarrow \lambda \alpha^{\rho}$ for some $\lambda, \rho \in \mathbb{R}$ with $\lambda \rho>0$, or $U: \alpha \leftrightarrow \log \alpha^{\prime \prime}$.

(ii) "The preference relation $\underline{\varepsilon}$ is a continuous strongly monotonic weak order, it satisfies the sure-thing principle, and has constant relative risk aversion".

Proof

Suppose (i). Then, for any $\mu>0, x \in \mathscr{C}^{n}$, for the expected utility EU, $\mathrm{EU}(\mu x)=\mu^{\rho} \mathrm{EU}(x)$ or $\mathrm{EU}(\mu x)=\mu+\mathrm{EU}(x)$. Constant relative risk aversion and all of (ii) follow straightforwardly from this. So we suppose (ii) and derive (i).

By strong monotonicity, every coordinate is "essential". By theorem 3 of Debreu [2], there exist continuous additive value functions $\left(V_{j}\right)_{j=1}^{n}$ for $£$. By strong monotonicity, every $V_{j}$ is strictly increasing. Define $V: \mathscr{C}^{n} \rightarrow \mathbb{R}, \phi: \mathscr{C} \rightarrow \mathbb{R}$, $W: G^{n} \rightarrow \mathbb{I} R$ by:

$$
V: x \rightarrow \sum V_{j}\left(x_{j}\right), \phi: \alpha \rightarrow V(\bar{\alpha}), W: x \rightarrow \phi^{-1} \circ V(x) .
$$

Then $V$ and $W$ represent $\xi ; W(\bar{\alpha})=\alpha ;[W(x)=\alpha \Rightarrow x \approx \bar{\alpha}]$, so $W$ assigns to each $x$ its "certainty equivalent". Also, $W(\mu x)=\mu W(x)$ for $\mu>0$ (W is "linearly homogeneous", so $V$ is "homothetic"). By Stehling ([8], theorem 2), or Eichhorn ([4], theorem 2.5.2), either:

$$
V: x \rightarrow \psi\left[\mu\left(\prod_{j=1}^{n} x_{i}^{p_{j}}\right)\right]
$$

for a continuous strictly increasing $\psi$, positive $\mu$, and nonzero $p_{1}, \ldots, p_{n}$

or:

$$
\text { that sum to one, }
$$

$$
V: x+\psi\left[\left(\sum_{j=1}^{n} \sigma_{j} x_{j}^{\rho}\right)^{1 / \rho}\right] \begin{aligned}
& \text { for a continuous strictly increasing } \psi, \\
& \text { positive } \sigma_{1}, \ldots, \sigma_{n} \text {, and nonzero } \rho .
\end{aligned}
$$

In the case of (3.4), $V$ is a strictly increasing transform of $x \rightarrow \Pi x_{j}^{p_{j}}$, and so, by taking logarithms, of $x \rightarrow \Sigma p_{j} \log \left(x_{j}\right)$. By strict increasingness of every $V_{j}$, every $p_{j}$ is positive. So indeed we have an SEU model for $\xi$, with $U: \alpha \rightarrow \log \alpha$. 
Next, suppose (3.5). First, assume $\rho>0$. Then $V$ is a strictly increasing transform of $\sum \sigma_{j} x_{j}^{\rho}$. We therefore have an SEU model for $\underline{\varepsilon}$, with $p_{j}:=\sigma_{j} / \Sigma_{i=1}^{n} \sigma_{i}$ for every $j$, and $U: \alpha \rightarrow \alpha^{\rho}$, so $\lambda=1$ in (i) above.

Finally, suppose (3.5), with $\rho<0$. Then $V$ is a strictly decreasing transform of $x \rightarrow \Sigma \sigma_{j} x_{j}^{\rho}$, so a strictly increasing transform of $x \rightarrow \Sigma \sigma_{j}\left(-x_{j}^{\rho}\right)$. We have an SEU model for $\underline{\xi}$, with $p_{j}:=\sigma_{j} / \Sigma_{i=1}^{n} \sigma_{i}$ for every $j$, and $U: \alpha \rightarrow-\left(\alpha^{\rho}\right)$, so in (i) above, $\lambda=-1$.

From this, we derive:

\section{THEOREM 3.5}

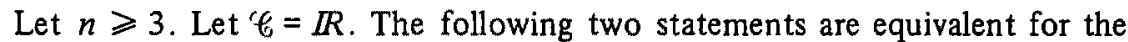
preference relation $\cong$ on ' $G^{n}$ :

(i) "There exists an SEU model $\left[\ell^{n}, \underline{\Sigma},\left(p_{j}\right)_{j=1}^{n}, U\right]$ for $\varepsilon$, with all $p_{j}>0$, and $U$ identity, or $U: \alpha \rightarrow \lambda \mathrm{e}^{\rho \alpha}$ for some $\lambda, \rho \in \mathbb{R}$ with $\lambda \rho>0^{\prime \prime}$.

(ii) "The preference relation is a continuous strongly monotonic weak order, it satisfies the sure-thing principle, and has constant absolute risk aversion".

Proof

Suppose (i). Then for any $\mu>0, x \in f^{n}$, the expected utility $\mathrm{EU}(x)$ has $\mathrm{EU}(x+\mu)=\mathrm{e}^{\rho \mu} \mathrm{EU}(x)$ or $\mathrm{EU}(x+\mu)=\mu+\mathrm{EU}(x)$. Constant absolute risk aversion and all of (ii) follow straightforwardly. So now we suppose (ii), and derive (i).

Define $L: \mathbb{R}_{++}^{n} \rightarrow \mathbb{R}^{n}$ by $L:\left(x_{1}, \ldots, x_{n}\right) \rightarrow\left(\log \left(x_{1}\right), \ldots, \log \left(x_{n}\right)\right)$, and define $\varliminf^{\prime}$ on $\mathbb{R}_{+}^{n}$ by $x \Xi^{\prime} y$ iff $L(x) \equiv L(y)$. Then it follows straightforwardly that $\varrho^{\prime}$ satisfies (ii) of theorem 3.4. We obtain, for all $x, y \in \mathbb{R}^{n}$ :

$$
x \underline{g} y \Leftrightarrow L^{-1}(x) \underline{\Xi}^{\prime} L^{-1}(y) \Leftrightarrow \sum p_{j} U\left(\mathrm{e}^{x_{j}}\right) \geqslant \sum p_{j} U\left(\mathrm{e}^{y_{j}}\right),
$$

with $U, p_{j}$, and also $\lambda, \rho$ as in (i) of theorem 3.4. This implies (i) of theorem 3.5.

Most probably, the last two theorems also hold for any interval $\ell_{2} \subset \mathbb{R}_{++}$, respectively, ' $\ell \subset \mathbb{R}$, but we do not know of a reference where the analogue of Stehling's [8] theorem, needed to prove this, is available.

We have not considered the case of $n=2$. One may replace $n \geqslant 2$ by $n=2$ in theorems 3.3 to 3.5 if one adds the so-called "Thomsen condition" (see Krantz et al. [5], definition 6.3) in (iii) of theorem 3.3 and (ii) of theorems 3.4 and 3.5. For $n=1$, the results are rather different; this concerns the case of decision making under certainty (and ordinal utility).

Uniqueness results in our theorems are standard, the utility function is always cardinal, and the subjective probabilities are uniquely determined. 


\section{Conclusion}

The approach by which the use of specified forms of subjective expected utility models is usually justified reflects the historical development of the theory of subjective expected utility maximization. Usually, in a first stage conditions are found, and justified, that are sufficient (and possibly necessary) for the existence of a subjective expected utility model without any further specification (for instance, the conditions in Savage [7]). Once this has been done, in a second stage behavioural conditions are found, and justified, which are sufficient (and possibly necessary) to obtain the desired specified form of the subjective expected utility model.

This paper has shown that a one-stage approach may lead to stronger results because assumptions on risk aversion, usually made in later stages, simplify the derivation in the first stage of the subjective expected utility model itself.

\section{Acknowledgement}

Many stylistic improvements have been given by an anonymous referee.

\section{References}

[1] E.W. Adams, R.IF. Fagot and R. Robinson, On the empirical status of axions in theories of fundamental measurement, Journal of Mathematical Psychology 7(1970)379.

[2] Q. Debreu, Topological methods in cardinal utility theory, in: Mathematical Methods in the Social Sciences, ed. K.J. Arrow, S. Karlin and P. Suppes (Stanford University Press, Stanford, 1960) pp. 16-26.

[3] G. Debreu and T.C. Koopmans, Additively decomposed quasi-convex functions, Math. Progr. 24(1982)1.

[4] W. Ejchhorn, Functional Equations in Economics (Addison-Wesley, Reading, MA, 1978).

[5] D.H. Krantz, R.D. Luce, P. Suppes and A. Tversky, Foundations of Measturement, Vol. I (Academic Press, New York, 1971).

[6] J.W. Pratt, Risk aversion in the small and in the large, Econometrica 32(1964)122.

[7] L.J. Savage, The Foundations of Statistics (Wiley, New York, 1954), 2nd ed: 1972.

[8] I: Stchling, Eine neue Charakterisierung der CD- und ACMS-Produktionsfunktionen, Operations Research-Verfahren 21 (1975)222.

[9] P.P. Wakker, Cardinal coordinate independence for expected utility, Journal of Mathematical Psychology 28(1984)1 10.

[10] P.P. Wakker, Continuous expected utility for arbitrary state spaces, Methods of Operations Research 50(1984)113.

[11] P.P. Wakker, Concave additively decomposable representing functions and risk aversion, in: Recent Developments in the Foundations of Utility and Risk Theory, cd. L. Daboni, A. Montesano and M. Lines (Reidel, Dord recht, 1986) pp. 249-262.

[12] P.P. Wakker, H.J.M. Peters and T.B.P.L. van Ricl, Comparisons of risk aversion, with an application to bargaining, Methods of Operations Research 54(1986)307. 